J. Appl. Cryst. (1974). 7, 181

\title{
Small-Angle X-ray Scattering of Native Hog Thyroglobulin
}

\author{
By H. Brumberger, J. Lipton, G. Dorfman and E. Nakano \\ Department of Chemistry, Syracuse University, Syracuse, N.Y. 13210, U.S.A.
}

\begin{abstract}
Thyroglobulin is a protein involved in the synthesis, storage and secretion of thyroid hormones. reported here are some small-angleX-1 ay measurements primarily concer ned with determination of its shape parameters in solution. The protein was fractionated on Sephadex G-200, and the portions corresponding to 'native' protein were collected and re-chromatographed on Sepharose 6-B; the protein samples were examined in phosphate buffer at $p \mathrm{H} 6 \cdot 9$. A radius of gyration of $64 \AA$, a particle volume of $\sim 1.7 \times 10^{6}$ $\AA^{3}$, and an anisotropy factor of 1.12 were found. Assuming a (reasonable) prolate ellipsoidal shape, one obtains an axial ratio of $2: 1$, in good agreement with recent electron micrographic results, and implying a high degree of solvation. Some details and problems connected with the SAXS measurements, in particular of the molecular weight, will be discussed.
\end{abstract}

J. Appl. Cryst. (1974). 7, 181

\section{X-ray Diffuse Scattering by Proteins in Solution. Consideration of Solvent Influence}

\author{
By B. A. Fedorov, O. B. Ptitsyn ANd L. A. Voronin \\ Institute of Protein Research, Academy of Sciences of the USSR, Poustchino, Moscow Region, USSR
}

(Received 8 September 1973; accepted 25 October 1973)

\begin{abstract}
Two methods of calculation of indicatrices of diffuse X-ray scattering by globular proteins in a medium with a non-zero electron density are described. The methods are used in calculation of curves of scattering by sperm whale native myoglobin in different solvents. The indicatrices obtained are close to the experimental. The nature of the so-called ' $10 \AA$ maximum', which is observed on the scattering curves of globular proteins in dense solvents as well as in concentrated protein solutions and protein gels, is discussed.
\end{abstract}

\section{Introduction}

Theoretical and experimental investigations of recent years have shown that a correct consideration of solvent influence is necessary for a valid interpretation of curves of X-ray diffuse scattering by polypeptides and proteins in solution in the range of comparatively large angles. Actually, a comparison of experimental indicatrices of scattering by an aqueous solution of sperm whale myoglobin (Mb) (Beeman, 1967; Kirste, Schulz \& Stuhrmann, 1969; Stuhrmann, 1970; Grigoryev, Volkova \& Ptitsyn, 1971b) with theoretical curves evaluated on the basis of the known coordinates of atoms (Watson, 1967; Voronin \& Fedorov, 1974), shows that beginning with $\sim 4^{\circ}$ (for $\mathrm{Cu} K \alpha$ emission) scattering indicatrices become essentially different. In their turn, the recently carried out analysis of theoretical curves of scattering by polypeptide chains in $\alpha$-helical and $\beta$-structural conformations (Voronin \& Fedorov, 1972; Voronin, Sarkisov, Kron \& Fedorov, 1973) and their comparison with experimental curves (Grigoryev, Volkova \& Ptitsyn, 1971a) have revealed considerable discrepancies between theory and experiment in the range of large angles. Naturally, the reason for this divergence is that the theoretical curves pertain to the molecule in vacuum, while the experimental ones pertain to the molecule surrounded by the solvent.

The main task, originating with the approach to the indicated problem, consists in the choice of a model of the solvent which can explain the experimental curves. Of course, this choice is determined by the factors which, in the first place, influence the picture of diffuse scattering at large angles: 1) the average electron density of the solvent; 2) the real structure of the solvent and 3) the change of this structure in the surrounding macromolecule. The consideration of just the first factor leads to the most simple model of the solvent convenient for calculation, i.e. a homogeneous continuum which is characterized only by the average electron density $\varrho_{0}$ (see, e.g., Hyman \& Vaughan, 1967). However, the possibility of applying this model $a$ priori raises doubts, since in the range of comparatively large angles $\left(10-25^{\circ}\right)$, which is of interest to us, the influence of the solvent structure (of a short-range order) must be rather strong. On the other hand, if only the consideration of all the three factors could 